Purdue University

Purdue e-Pubs

CTRC Research Publications

Cooling Technologies Research Center

2004

\title{
A Two-Temperature Model for the Analysis of Passive Thermal Control Systems
}

S. Krishnan

J. Y. Murthy

S V. Garimella

Purdue University, sureshg@purdue.edu

Follow this and additional works at: http://docs.lib.purdue.edu/coolingpubs

Krishnan, S.; Murthy, J. Y.; and Garimella, S V., "A Two-Temperature Model for the Analysis of Passive Thermal Control Systems" (2004). CTRC Research Publications. Paper 275.

http://dx.doi.org/10.1115/1.1773194

This document has been made available through Purdue e-Pubs, a service of the Purdue University Libraries. Please contact epubs@purdue.edu for additional information. 


\section{A Two-Temperature Model for the Analysis of Passive Thermal Control Systems}

\section{Shankar Krishnan} Jayathi Y. Murthy Suresh V. Garimella e-mail: sureshg@ecn.purdue.edu

Cooling Technologies Research Center, School of Mechanical Engineering, Purdue University, West Lafayette, IN 47907-2088
Passive control of steady and unsteady thermal loads using effective thermal conductivity enhancers, such as metal foams, internal fins and metal filler particles, is being explored for a variety of electronics applications. The interstices are filled with air, phase change materials, or other fluids. Local thermal equilibrium between the solid filler and the matrix is not ensured in such systems since their thermal diffusivities are frequently very different. The use of a single volume-averaged energy equation for both the phases cannot be justified in such situations. A two-medium approach is used in the present work to account for the local thermal non-equilibrium. Separate energy equations are written for the solid and fluid respectively, and are closed using a steady-state interphase heat transfer coefficient between the two phases. A general momentum equation which includes the Brinkman-Forchheimer extension to Darcy flow is employed. The resulting equations are solved implicitly using a fully transient method on fixed orthogonal co-located finite volumes. Unsteady natural convection in a metal-foam filled cavity is computed. The influence of various parameters such as the ratios of solid-to-fluid thermal conductivities and heat capacities, Rayleigh number, Prandtl number and Darcy number on the thermal and flow fields is investigated. The results illustrate that local thermal equilibrium is not assured, either during the transient or at steady state for the range of parameters considered. Furthermore, even if the steady-state solid-to-fluid temperature differences are small, large temperature differences are seen during the unsteady response. [DOI: $10.1115 / 1.1773194]$

Keywords: Enhancement, Heat Transfer, Natural Convection, Non-Equilibrium, Porous Media

\section{Introduction}

Thermal management of electronics is becoming increasingly challenging as chip-level heat fluxes increase at a rapid rate. Cooling can be achieved either by using conventional techniques that utilize active components like fans, or with passive techniques such as phase change thermal storage units and heat pipes. In many emerging cooling designs, thermal conductivity enhancers such as metallic foams, internal fins and metallic particles are used. The interstices contain either fluid or air. In thermal energy storage applications, for example, phase change materials (PCMs) are used. These materials are particularly attractive for transient applications where the heat generation experiences frequent short spikes in its magnitude. In addition to a high latent heat of melting, phase change materials used in electronics cooling must have high thermal diffusivity to quickly conduct away the dissipated heat into the bulk of the PCM. Though typical PCMs in popular use (e.g., paraffins) have very high latent heats of melting (order of $10^{5} \mathrm{~J} / \mathrm{kg}$ or more) [1] and isobaric specific heat capacities (order of $1000 \mathrm{~J} / \mathrm{kg} \mathrm{K}$ or more) [1], they suffer from very low thermal conductivities (order of $1 \mathrm{~W} / \mathrm{mK}$ or less). Further discussion of the characteristics of solid/liquid and liquid/vapor PCMs is available in $[2,3]$. It is therefore necessary to increase the effective thermal conductivity of the PCM through the use of internal fins, foams or filler particles. In high power electronics applications, metal foams with high porosity have been proposed to provide increased surface area for passive single-phase heat transfer.

In general, metal foams are mathematically modeled using the technique of volume-averaging owing to the complexity involved in modeling the metal foam and saturating fluid separately. Hunt

Contributed by the Heat Transfer Division for publication in the JOURNAL OF HEAT TRANSFER. Manuscript received by the Heat Transfer Division June 2, 2003; revision received March 24, 2004. Associate Editor: K. S. Ball. and Tien [4] explored forced convection in metal foams saturated with water. They studied the effects of thermal dispersion on forced convection using a single energy equation assuming a local thermal equilibrium between the metal foam and fluid phase.

Amiri and Vafai $[5,6]$ used a semi-heuristic model to account for local thermal nonequilibrium for steady and transient forced convective flows through a bed of spherical particles. They explored the importance of non-Darcian terms and thermal dispersion effects on thermal and flow characteristics. Error maps were introduced to quantify the results. The solid-to-fluid thermal conductivity ratio was found to have a profound influence on the local thermal equilibrium. Lee and Vafai [7] performed an analytical study of forced convective flows in a porous bed. An electrical network was developed to represent the heat transfer through the porous medium. The validity of a single volume-averaged energy equation was discussed based on the solid and fluid temperature differentials obtained from a two-energy equation model. The error in using the one-equation model was found to increase with a decrease in the ratio of effective conductivity of the fluid to that of the solid and with a decrease in Biot number based on the interstitial heat transfer coefficient. Lu et al. [8] developed an analytical model for the convection heat transport through metal foams. Simplifying assumptions were made and guidelines for optimum foam structures were proposed for maximum heat transfer for a given power input. Minkowycz et al. [9] performed a theoretical study to analyze the validity of assuming local thermal equilibrium in heat transfer through a porous fluidized bed. It was shown that local thermal equilibrium depends on the size of the porous layer, pore size, interstitial heat transfer coefficient and thermophysical properties. Calmidi and Mahajan [10] performed an experimental investigation of forced convection in metal foams with air and water as fluid phases. An empirical correlation was also reported. Alazmi and Vafai [11] analyzed a variety of porous me- 


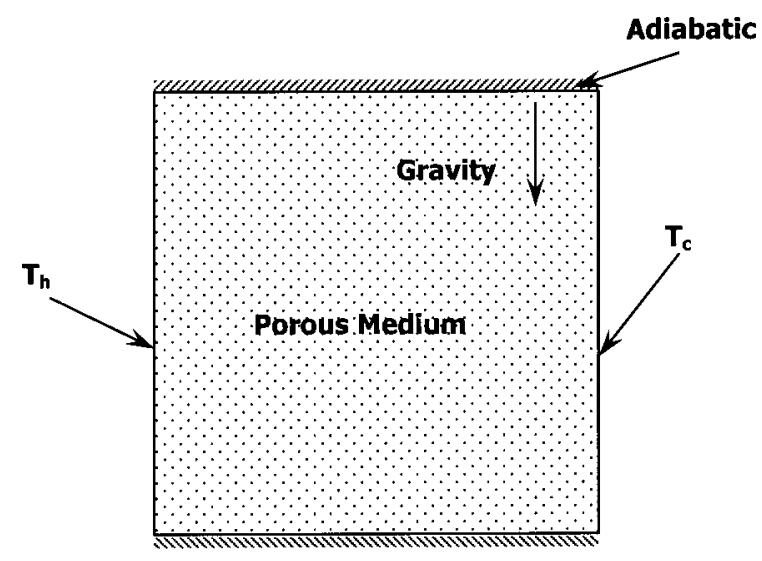

Fig. 1 Schematic diagram of the problem considered

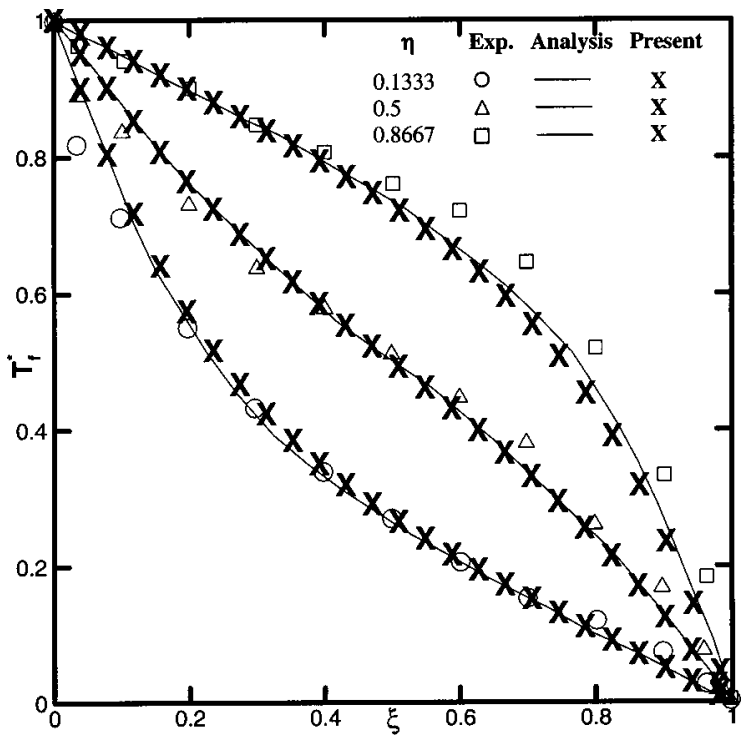

Fig. 2 Comparison of the present work $(X)$ with experimental and numerical predictions of Beckermann and Viskanta [20]

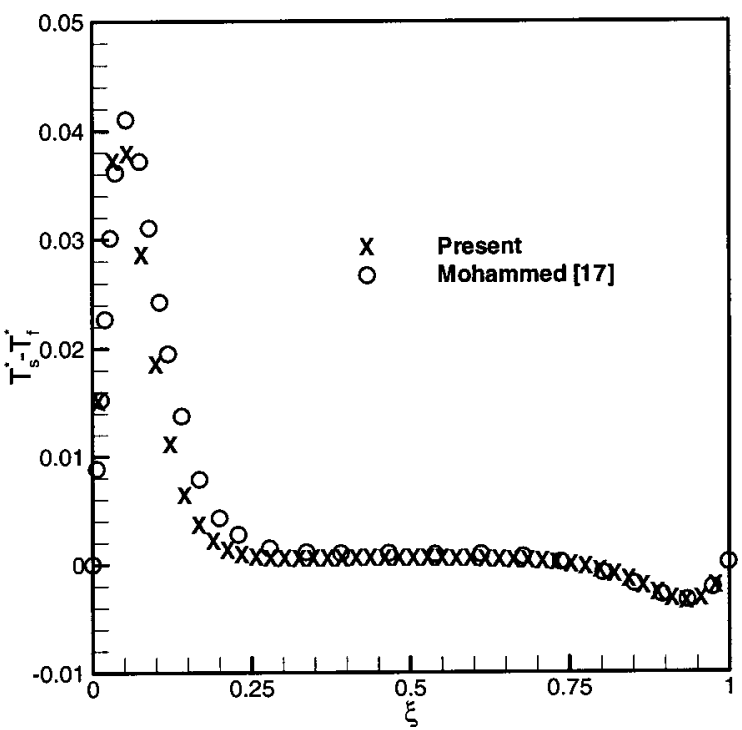

Fig. 3 Comparison of the predicted temperature difference between solid and fluid phases $(X)$ with the numerical predictions of Mohammed [18] $(O)$ at $\boldsymbol{\eta}=0.25$ for $\operatorname{Pr}=1$ and thermal conductivity ratio $=1.0$

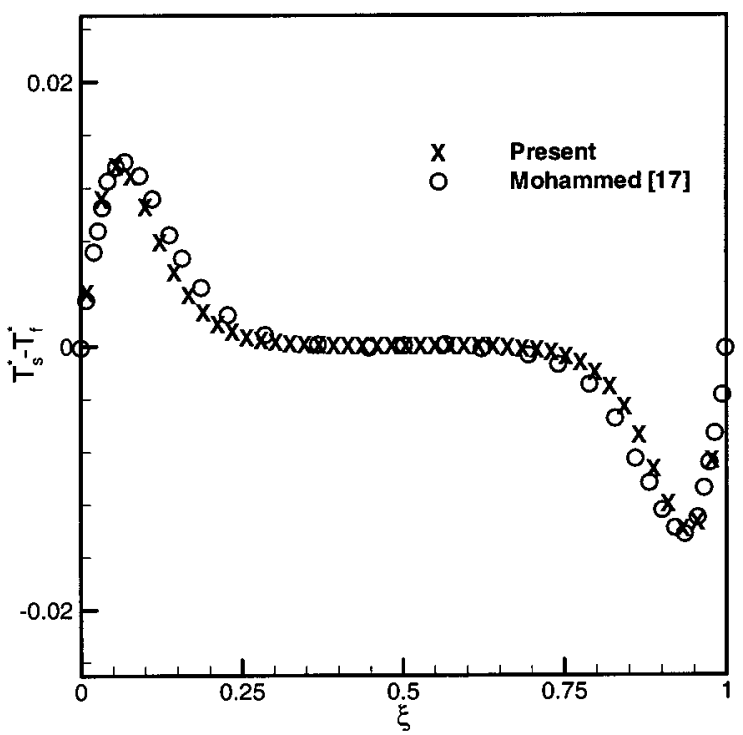

Fig. 4 Comparison of the predicted temperature difference between solid and fluid phases $(X)$ with the numerical predictions of Mohammed [18] $(\bigcirc)$ at $\boldsymbol{\eta}=\mathbf{0 . 5}$ for $\mathrm{Pr}=1$ and thermal conductivity ratio $=1.0$

dia transport models for forced convection in porous beds. The effects of variations in existing semi-heuristic models, effects of porosity, thermal dispersion and local thermal equilibrium were reported. Calmidi and Mahajan [12] reported an experimental and numerical study of forced convection in metal foams under local thermal non-equilibrium conditions. Hwang et al. [13] performed experiments on metal foams. They measured the interstitial convective heat transfer and frictional drag for forced convective flows using a transient single-blow technique. Empirical correlations for interstitial heat transfer coefficient were reported. Further details of the thermal non-equilibrium modeling of forced convection can be obtained from [14].

Natural convection in porous media is also encountered in many applications $[15,16]$. However, while many studies have

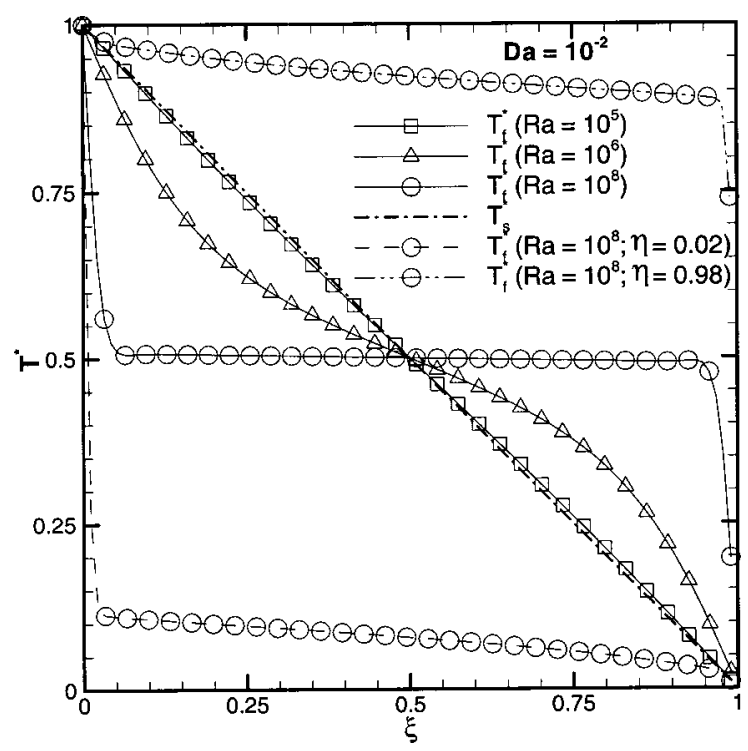

Fig. 5 Spatial variation of solid and fluid temperature distribution for zero inter-phase heat transfer coefficient $\left(\mathrm{Nu}_{f}=\mathbf{0}\right)$. For all Rayleigh numbers the solid temperature distribution is a straight line. 


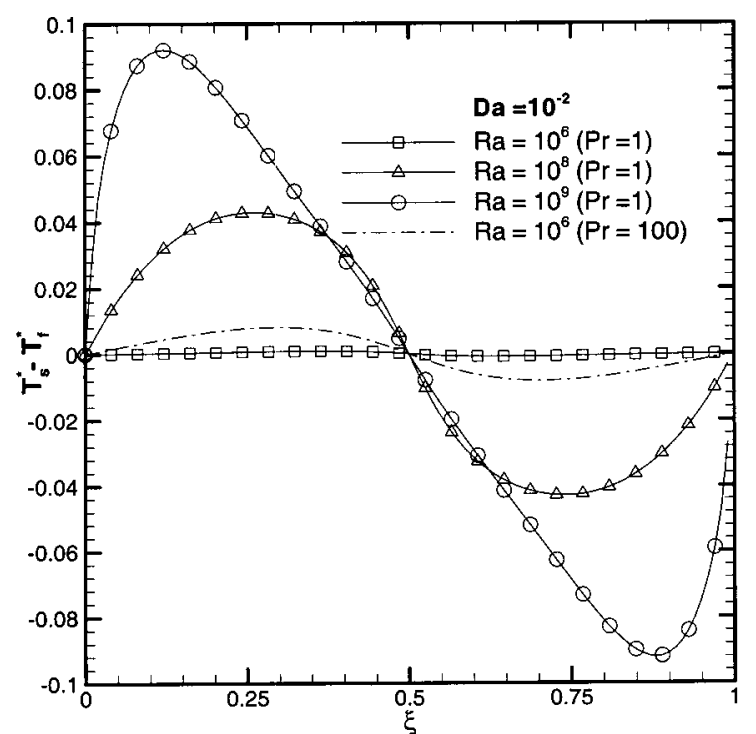

(a)

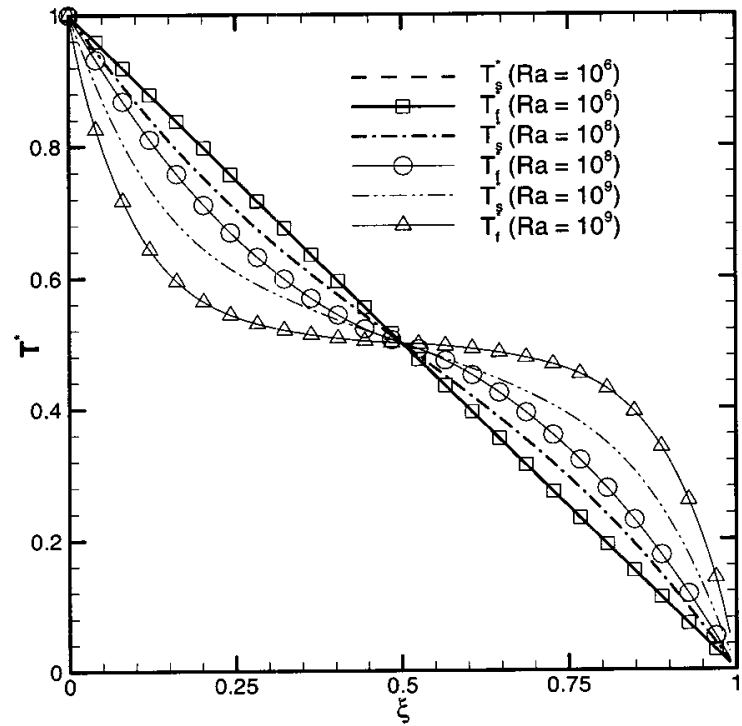

(b)

Fig. 6 Predicted temperature variation at steady-state for various Rayleigh numbers at the mid-height of the domain $(\eta=0.5)$ : (a) solid-to-fluid temperature difference, and (b) solid (broken line) and fluid (solid lines with symbols) temperature distributions

investigated forced convective flow through porous media under local non-equilibrium thermal conditions, fewer have considered natural convection. Deiber and Bortolozzi [17] performed a natural convection study in a vertical porous annulus composed of spheres without assuming local thermal equilibrium to exist between the solid matrix and saturating fluid. They reported that a two-energy equation model should be used at high Rayleigh and Darcy numbers. Mohammed [18] performed a parametric study for natural convection in a porous enclosure under steady-state conditions. The effects of varying the Darcy, Rayleigh, and Prandtl numbers and the solid-to-fluid thermal conductivity ratio on the temperature differentials between solid and fluid were reported. Rees and Pop [19] also reported the existence of nonequilibrium conditions for steady-state flows. Beckermann and Viskanta [20] experimentally and numerically analyzed natural convection inside a porous enclosure with a local thermal equilibrium assumption. Recently, Phanikumar and Mahajan [21] reported numerical and experimental results for natural convective

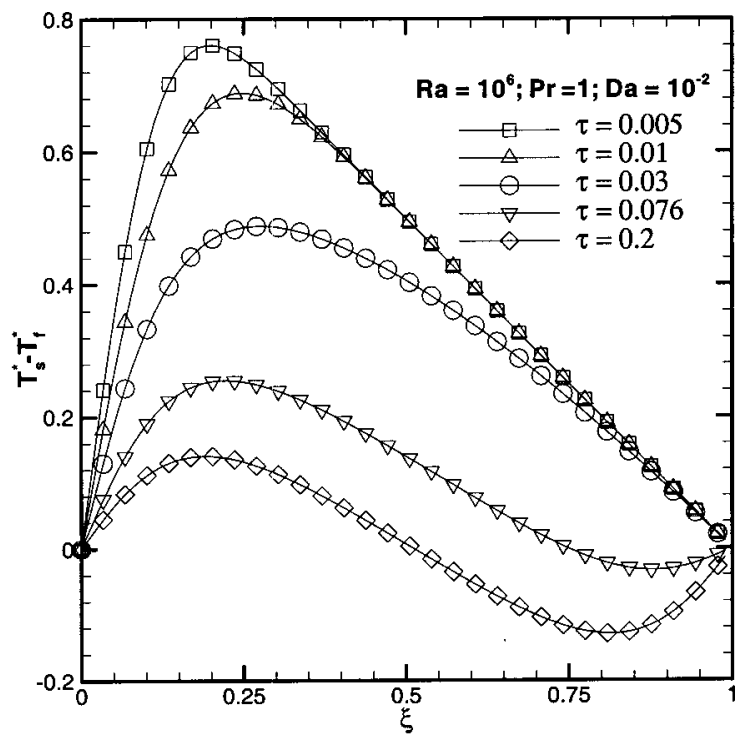

(a)

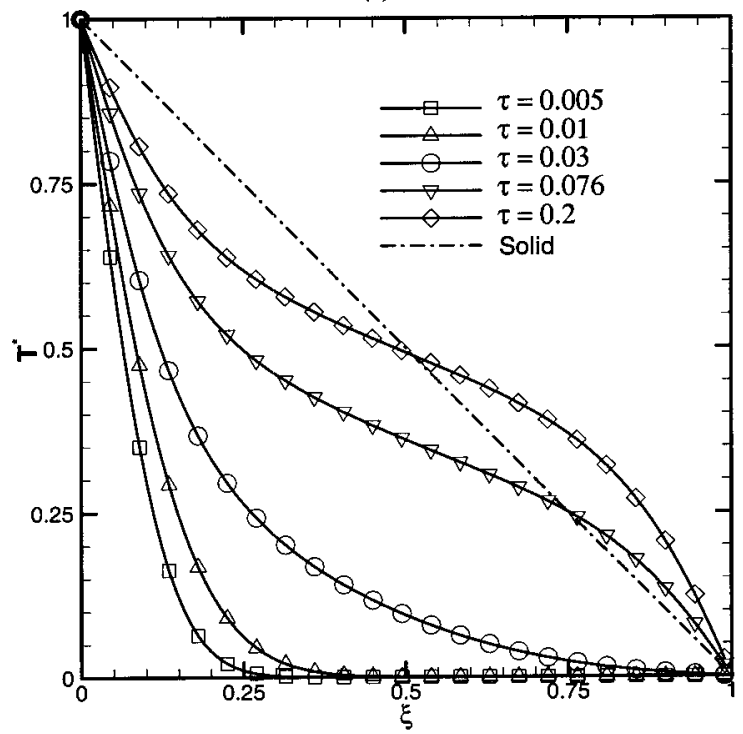

(b)

Fig. 7 Predicted temporal evolution of thermal field for Ra $=10^{6}, \mathrm{Nu}_{f}=0, \mathrm{Pr}=1$, and $\mathrm{Da}=10^{-2}$ at the mid-height of the domain $(\eta=0.5)$ : (a) solid-to-fluid temperature difference, and $(b)$ solid (broken line) and fluid (solid lines with symbols) temperature distribution. Solid (broken line) reaches a steady state very fast.

flow in a rectangular domain partially filled with a porous medium (metal foam) and heated from below. Heat transfer enhancement due to the presence of the foam against that with no foam was studied. Effects of thermal dispersion and the Darcy number on the heat transfer were explored. They concluded that a local thermal nonequilibrium model better describes the underlying heat transfer phenomena in metal foams.

The present work undertakes a parametric analysis of natural convection inside enclosures containing metal foams for the transient thermal management of electronics. Only single-phase flows without phase change are considered to elucidate the basic physics. The paper examines the transient effects of different parameters including the Rayleigh, Darcy and Prandtl numbers, solidto-fluid thermal conductivities, and the Nusselt number for interphase heat transfer, on the temperature differentials between 


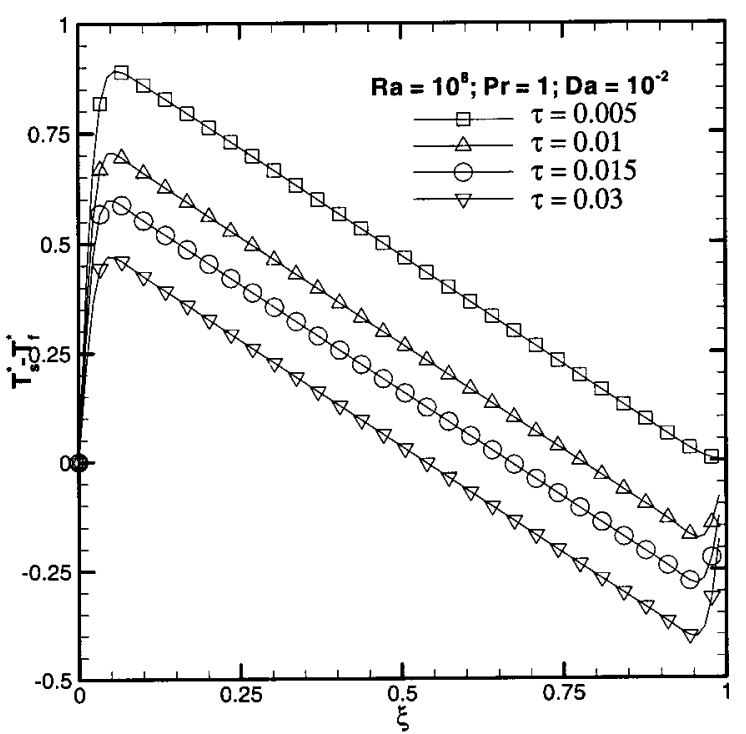

(a)

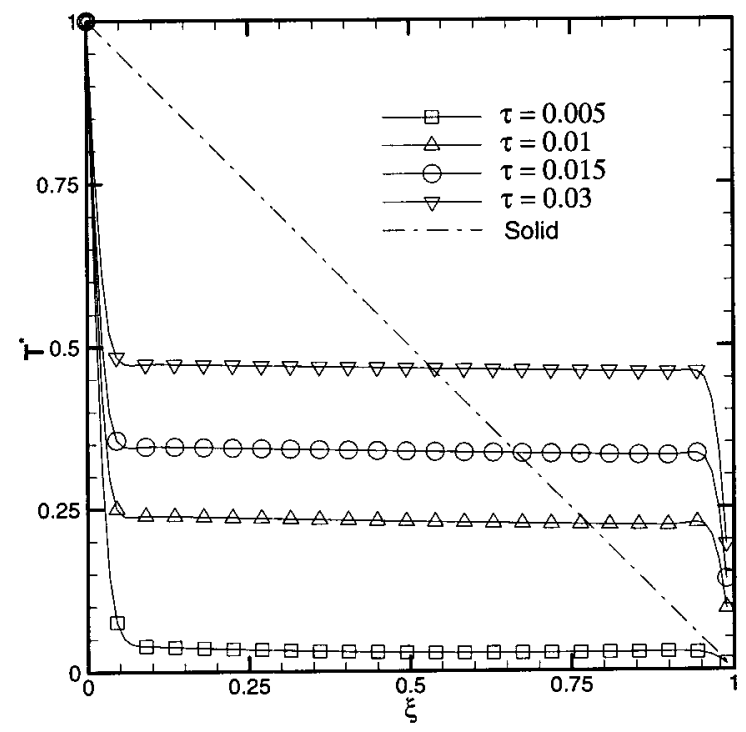

(b)

Fig. 8 Predicted temporal evolution of thermal field for Ra $=10^{8}, \mathrm{Nu}_{f}=0, \mathrm{Pr}=1$, and $\mathrm{Da}=10^{-2}$ at the mid-height of the domain $(\eta=0.5)$ : (a) solid-to-fluid temperature difference, and $(b)$ solid (broken line) and fluid (solid lines with symbols) temperature distribution. Solid (broken line) reaches a steady state very fast.

the solid and fluid under local thermal non-equilibrium conditions. The physics governing the existence of thermal non-equilibrium during the transient are explored.

\section{Mathematical Formulation and Numerical Modeling}

A schematic of the problem considered is shown in Fig. 1. A porous medium saturated with fluid is contained in a square enclosure of side $H$. The left and right walls are held at constant temperatures $T_{h}$ and $T_{c}$, respectively. The top and bottom walls are adiabatic. The fluid and solid media are at equilibrium initially, and at temperature $T_{c}$. At $\tau=0$, the temperature of the left wall is raised to $T_{h}$. The objective of the work is to examine the temporal evolution of the solid and fluid temperature fields. The liquid is assumed to be incompressible and Newtonian. The momentum equations include the Brinkman's term and Forchheimer's extension to Darcy flow. The solid is assumed to be isotropic and rigid. Thermophysical properties of the solid and fluid

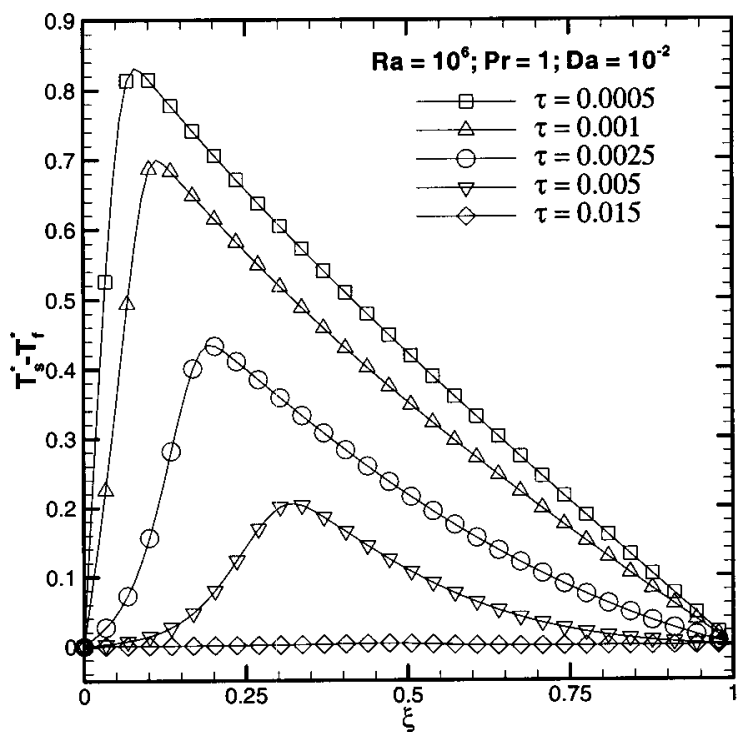

(a)

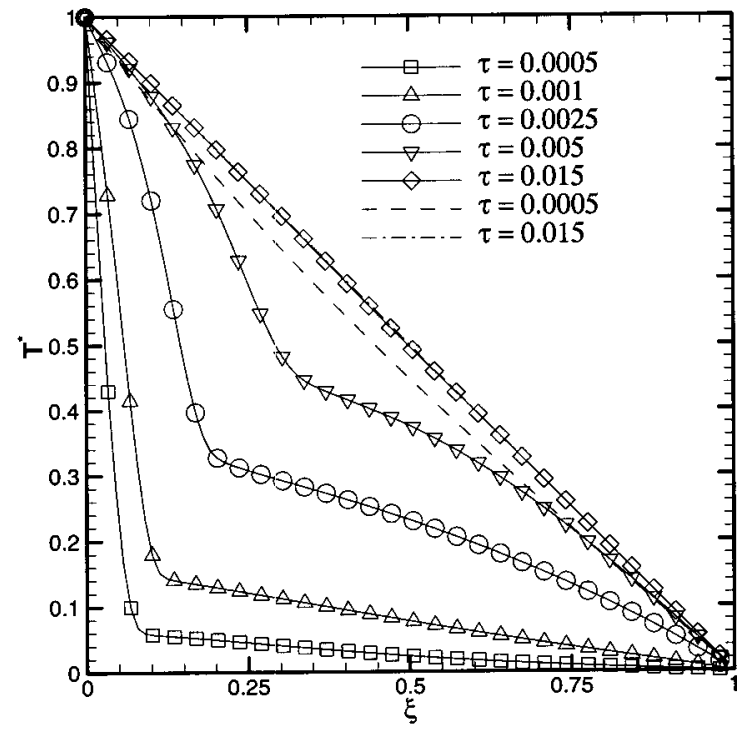

(b)

Fig. 9 Predicted temporal evolution of thermal field for $\mathbf{R a}$ $=10^{6}, \mathrm{Nu}_{f} \neq 0, \mathrm{Pr}=1$, and $\mathrm{Da}=10^{-2}$ at the mid-height of the domain $(\eta=0.5)$ : (a) solid-to-fluid temperature difference, and $(b)$ solid (broken line) and fluid (solid lines with symbols) temperature distribution

phases are assumed to remain constant and isotropic over the range of temperatures considered. The Boussinesq approximation is invoked in the fluid phase. Dispersion effects are neglected. Using the dimensionless variables

$$
\begin{gathered}
\xi=\frac{x}{H} ; \quad \eta=\frac{y}{H} ; \quad \tau=\frac{t \alpha_{f}}{H^{2}} ; \quad \mathbf{U}=\frac{u H}{\alpha_{f}} ; \quad P=\frac{p H^{2}}{\rho_{f} v_{f} \alpha_{f}} \\
T_{s}^{*}=\frac{\left(T_{s}-T_{c}\right)}{\left(T_{h}-T_{c}\right)} ; \quad T_{f}^{*}=\frac{\left(T_{f}-T_{c}\right)}{\left(T_{h}-T_{c}\right)}
\end{gathered}
$$

the dimensionless volume-averaged continuity and momentum equations [20] in the fluid phase are

$$
\nabla \cdot \mathbf{U}=0
$$


Table 1 (a) Predicted times for solid and fluid to reach steady state for various Rayleigh, Prandtl and Darcy numbers for $\mathrm{Nu}_{f}=0$, and (b) predicted solid-to-fluid temperature differences at steady state

\begin{tabular}{|c|c|c|c|c|}
\hline Darcy Number & Prandtl Number & Rayleigh Number & $\begin{array}{l}\text { Time }{ }^{1} \text { for solid to } \\
\text { reach steady state }\end{array}$ & $\begin{array}{l}\text { Time }{ }^{1} \text { for fluid to } \\
\text { reach steady state }\end{array}$ \\
\hline \multirow[t]{6}{*}{$10^{-2}$} & 1 & $10^{5}$ & 0.00055 & 0.45 \\
\hline & & $10^{6}$ & 0.00055 & 0.2 \\
\hline & & $10^{8}$ & 0.00055 & 0.03 \\
\hline & & $10^{9}$ & 0.00055 & 0.015 \\
\hline & 100 & $10^{6}$ & 0.00055 & 0.1925 \\
\hline & & $10^{8}$ & 0.00055 & 0.0275 \\
\hline \multirow[t]{4}{*}{$10^{-3}$} & 1 & $10^{6}$ & 0.00055 & 0.55 \\
\hline & & $10^{8}$ & 0.00055 & 0.175 \\
\hline & 100 & $10^{6}$ & 0.00055 & 0.58 \\
\hline & & $10^{8}$ & 0.00055 & 0.171 \\
\hline
\end{tabular}

(a)

\begin{tabular}{ccccc} 
Darcy Number & Prandtl Number & Rayleigh Number & $\begin{array}{c}\left(\mathrm{T}_{\mathrm{s}}-\mathrm{T}_{\mathrm{f}}\right)_{\max } \\
\text { at } \eta=0.5\end{array}$ & $\left(\mathrm{~T}_{\mathrm{s}}-\mathrm{T}_{\mathrm{f}}\right)_{\max }$ \\
\hline $10^{-2}$ & 1 & $10^{5}$ & 0.01 & 0.15 \\
& & $10^{6}$ & 0.13 & 0.57 \\
& 100 & $10^{8}$ & 0.44 & 0.86 \\
& 10 & 0.48 & 0.91 \\
& 1 & $10^{6}$ & 0.13 & 0.575 \\
$10^{-3}$ & 100 & $10^{8}$ & 0.44 & 0.86 \\
& 100 & $10^{6}$ & 0.0002 & 0.017 \\
& & $10^{8}$ & 0.13 & 0.61 \\
& & $10^{6}$ & 0.00015 & 0.0168 \\
& & $10^{8}$ & 0.128 & 0.61
\end{tabular}

(b)

${ }^{1}$ Nondimensional time.

$$
\begin{aligned}
\frac{1}{\operatorname{Pr}}\left(\frac{1}{\varepsilon} \frac{\partial U}{\partial \tau}+\frac{1}{\varepsilon^{2}}(\mathbf{U} \cdot \nabla) \mathbf{U}\right)= & -\nabla \mathbf{P}+\frac{1}{\varepsilon} \nabla^{2} \mathbf{U}-\left(\frac{1}{\mathrm{Da}^{2}}\right. \\
& \left.+\frac{F}{\mathrm{Pr} \cdot \mathrm{Da}}|\mathbf{U}|\right) \mathbf{U}+\mathrm{Ra} \cdot T_{f}^{*}
\end{aligned}
$$

The governing intrinsic phase-averaged energy equations are written for the solid and fluid phases separately. These macroscopic continuum equations for solid and fluid are valid at every point in the domain. The two energy equations can be closed either using an unsteady closure [22] or a steady-state closure [5]. A steadystate closure has been found to be sufficient for most problems [22], and is used in this work. The dimensionless energy equations are

$$
\begin{gathered}
\Omega \frac{\partial T_{s}^{*}}{\partial \tau}=\lambda \nabla^{2} T_{s}^{*}-\frac{(\mathrm{Nu})_{f}}{(1-\varepsilon)}\left(T_{s}^{*}-T_{f}^{*}\right) \\
\frac{\partial T_{f}^{*}}{\partial \tau}+\frac{1}{\varepsilon}(\mathbf{U} \cdot \nabla) T_{f}^{*}=\nabla^{2} T_{f}^{*}-\frac{(\mathrm{Nu})_{f}}{\varepsilon}\left(T_{f}^{*}-T_{s}^{*}\right)
\end{gathered}
$$

The dimensionless parameters governing the flow and heat transfer are

$$
\begin{gathered}
\operatorname{Pr}=\frac{v_{f}}{\alpha_{f}} ; \quad \mathrm{Ra}=\frac{g \beta_{f} H^{3}\left(T_{h}-T_{c}\right)}{\alpha_{f} v_{f}} ; \quad \mathrm{Da}=\frac{\sqrt{K}}{H} ; \quad(\mathrm{Nu})_{f}=\frac{h_{v} H^{2}}{k_{f}} \\
\lambda=\frac{k_{s}}{k_{f}} ; \quad \Omega=\frac{\left(\rho C_{p}\right)_{s}}{\left(\rho C_{p}\right)_{f}} ; \quad \delta=\frac{\mathrm{d}}{\mathrm{H}} \\
F=0.068 \quad(\text { for metal foams) } \\
F=\frac{1.75}{\sqrt{150 \varepsilon^{3}}} \quad \text { (for flow over spherical particles) }
\end{gathered}
$$

Two different volumetric heat transfer coefficients are used. For forced convection through a packed bed of spheres $[16,23]$ the expression is

$$
(\mathrm{Nu})_{p}=\frac{h_{v} d^{2}}{k_{f}}=6(1-\varepsilon)\left[2+1.1 \operatorname{Pr}^{1 / 3}(\mathrm{Re})^{0.6}\right]
$$

This correlation is valid up to Re of 8500 . For forced flow through metal foams, the following correlation is used

$$
(\mathrm{Nu})_{p}=\frac{h_{v} d^{2}}{k_{f}}=0.376(\mathrm{Re})^{0.644}(\mathrm{Pr})^{0.37}
$$

It is important to note the limitations of Eq. (6) in modeling interphase heat transfer. The correlation in [13] was developed for metal foams in air, for a Reynolds number $\operatorname{Re}^{*}(=u L / v, L$ being the test-section length) in the range $1900<\mathrm{Re}^{*}<7900$. To use it for typical PCMs, a scaling factor for Prandtl number similar to that in Phanikumar and Mahajan [21] was included. Further, for high $\mathrm{Pr}$, the flow in the core of the domain is of relatively low velocity, and the Reynolds number may be far lower than the lower Re* limit of Eq. (6); it is also clear that Eq. (6) does not correctly recover the conduction $\left(\mathrm{Re}^{*}=0\right)$ limit. To the authors' knowledge, there are no available correlations in the literature describing the inter-phase heat transfer coefficient in the highPrandtl number and low-Reynolds number limit. Therefore, Eq. (6) is used despite these limitations.

The volumetric heat transfer coefficient in the above equations can be expressed in terms of the inter-phase heat transfer coefficient as

$$
h_{v}=h_{s f} a_{s f}
$$

In Eq. (7), $a_{s f}$ is the specific surface area. These correlations are expected to be approximately valid for natural convection as well. The Reynolds number in Eqs. (5) and (6) was interpreted as the local Reynolds number, defined based on the local mean velocity 


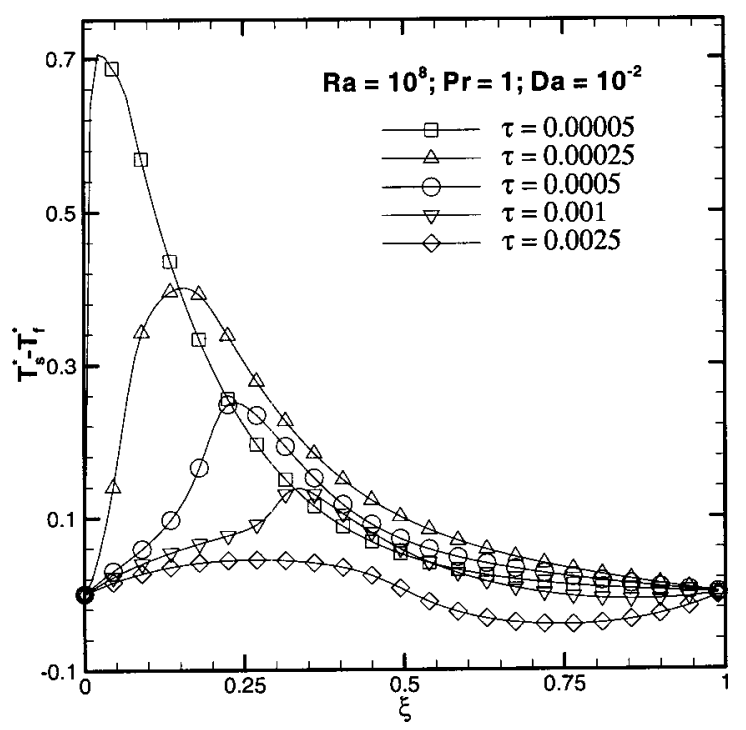

(a)

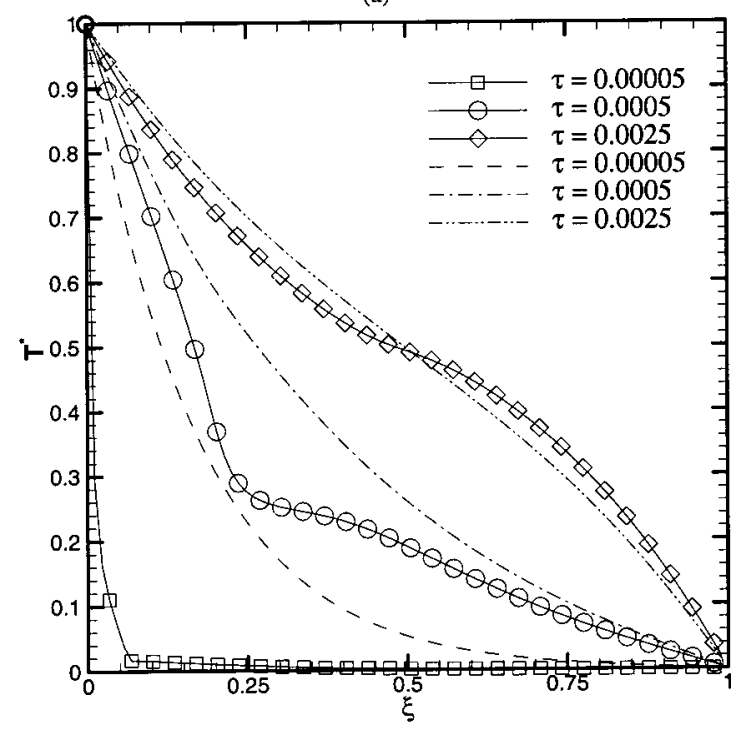

(b)

Fig. 10 Predicted temporal evolution of thermal field for $\mathbf{R a}$ $=10^{8}, \mathrm{Nu}_{f} \neq 0, \mathrm{Pr}=1$, and $\mathrm{Da}=10^{-2}$ at the mid-height of the domain $(\eta=0.5)$ : (a) solid-to-fluid temperature difference, and (b) solid (broken line) and fluid (solid lines with symbols) temperature distribution

$\left(u_{\text {mean }} d / \nu\right)$. The relations for permeability and inertial coefficient used for spherical particles can be obtained from [5,6]. For metal foams, a porosity of 0.8 is used and the inertial coefficient is 0.068 [13].

The computational domain is discretized into finite volumes using an orthogonal mesh. Pressure, velocity and the two temperatures are stored at the cell centroids. A central differencing scheme with a deferred correction $[24,25]$ is used for convective fluxes. A central differencing scheme is used for discretizing diffusive fluxes. A second order Euler implicit (three time level) scheme is used for the transient terms. The SIMPLE algorithm is employed for obtaining the velocity fields. The linearized systems of equations are solved using the strongly implicit procedure (SIP) [25]. Additional details about the numerical approach are available in $[2,26]$. The calculations are terminated when the residual has dropped at least below $10^{-6}$ for all governing equations.

\section{Code Validation}

Because experimental results are not available in the literature for natural convection inside porous metal foams, the code was validated against studies on convection in a packed bed of spheres. Beckermann and Viskanta [20] performed an experimental and numerical study of natural convection in a porous enclosure comprising spherical (glass) particles with gallium as the interstitial fluid. A fixed porosity of 0.385 was considered. Predictions from the present work are compared to those in [20] in Fig. 2 at different vertical ( $\eta$-direction) locations. In the current predictions, Eq. (5) was used to represent the inter-phase heat transfer coefficient. The predicted thermal fields agree well with the numerical predictions in [20] and also show satisfactory agreement with the experimental measurements [20].

Predictions from the present model are also compared in Figs. 3 and 4 with those of Mohammed [18] who also employed a twotemperature model for the analysis of natural convection in a packed bed of spheres. The comparison is shown for the case of Prandtl number $=1.0$, and the agreement is seen to be excellent.

Grid and time-step independence for the mesh and time-step sizes used in the present simulations was also established. A Rayleigh number of $10^{8}$, a Darcy number of $10^{-3}$ and a Prandtl number of 1 were used for this set of calculations. Grid independence was tested using three different non-uniform grids, $46 \times 46$ (mesh \#1), $92 \times 92$ (mesh \#2) and $182 \times 182$ (mesh \#3). For mesh \#1, a deviation of $6.48 \%$ in the maximum solid-to-fluid temperature difference was observed with respect to mesh \#3, with this deviation between mesh \#2 and mesh \#3 reducing to $0.33 \%$. Therefore, mesh \#2 $(92 \times 92)$ was chosen for all the calculations in this study. For establishing time-step independence, three different time step values, $1.25 \times 10^{-4}, 2.5 \times 10^{-4}$ and $5 \times 10^{-4}$ were evaluated with the $92 \times 92$ mesh. The deviation in the results for the maximum solid-to-fluid temperature difference compared to the smallest time step was less than $0.01 \%$ for both larger time steps. Since refinement in the time-step did not produce discernible improvements in accuracy, a nondimensional time-step value of $2.5 \times 10^{-4}$ was used for most of the computations in this work.

\section{Results and Discussion}

Passive thermal storage units used in electronics cooling would feature solid-to-fluid thermal conductivity ratios $(\lambda)$ in the range of $10^{2}$ to $10^{5}$ and Prandtl numbers of 1 to 100 . The porosity of the metal foam considered in this study is held constant at 0.8 with a pore size corresponding to $d / H=0.0135$. The ratio of the average ligament diameter of the foam to the mean cell size of the foam is 0.1875 with average ligament diameter being equal to $0.36 \mathrm{~mm}$ [13]. In the range of thermal conductivity ratios of interest $(\lambda$ $\sim 10^{3}-10^{5}$ ), the results did not vary much with $\lambda$, and hence a value of $10^{3}$ was used for all cases.

Steady State. The case of zero inter-phase heat transfer $\left(\mathrm{Nu}_{f}=0\right)$ is first considered, in which there is no thermal interaction between the solid and fluid phases. Figure 5 shows the variation of solid and fluid temperature profiles for different Rayleigh numbers at mid-height $(\eta=0.5)$; the fluid temperature variation at $\eta=0.02$ and $\eta=0.98$ for $\mathrm{Ra}=10^{8}$ is also shown for contrast. The solid profile is expected to be a straight line and is independent of the Prandtl number and $\eta$ location. The fluid profile is also a straight line if the Rayleigh number is low and, as a result, the solid-to-fluid temperature difference $\Delta T^{*} \sim O(0)$ everywhere in the domain. As the Rayleigh number increases the fluid temperature profile deviates from the solid temperature profile as thermal boundary layers develops near the heated and cooled walls. The thickness of the boundary layer scales as $\sim\left(\mathrm{RaDa}^{2}\right)^{-1 / 4}$ [27]. It can be seen from Fig. 5 that the numerical predictions agree with the scaling analysis. For example, for $\mathrm{Ra}=10^{8}$ and $\mathrm{Da}=10^{-2}$ the numerically predicted boundary layer thickness is approximately 0.09 and that from scaling analysis is 0.1 . As the Rayleigh number increases, the thermal boundary layer in the fluid is increasingly confined to a thin layer near the wall, with the core being at a temperature $T_{f}^{*} \sim 0.5$. Thus, the geometric center of the cavity is a point of zero temperature difference between solid and fluid. The 
Table 2 (a) Predicted times for solid and fluid to reach steady state for various Rayleigh, Prandtl and Darcy numbers for $\mathrm{Nu}_{f} \neq 0$, and (b) predicted solid-to-fluid temperature differences at steady state

\begin{tabular}{|c|c|c|c|c|}
\hline Darcy Number & Prandtl Number & Rayleigh Number & $\begin{array}{l}\text { Time }{ }^{1} \text { for solid to } \\
\text { reach steady state }\end{array}$ & $\begin{array}{l}\text { Time }{ }^{1} \text { for fluid to } \\
\text { reach steady state }\end{array}$ \\
\hline \multirow[t]{4}{*}{$10^{-2}$} & 1 & $10^{6}$ & 0.0075 & 0.00935 \\
\hline & & $10^{8}$ & 0.0023 & 0.00235 \\
\hline & 100 & $10^{6}$ & 0.015 & 0.0225 \\
\hline & & $10^{8}$ & 0.0027 & 0.00275 \\
\hline \multirow[t]{5}{*}{$10^{-3}$} & 1 & $10^{6}$ & 0.02345 & 0.0929 \\
\hline & & $10^{8}$ & 0.00735 & 0.0086 \\
\hline & 10 & $10^{6}$ & 0.0125 & 0.15 \\
\hline & 100 & $10^{6}$ & 0.00055 & 0.2245 \\
\hline & & $\begin{array}{l}10^{8} \\
\text { (a) }\end{array}$ & 0.01475 & 0.02175 \\
\hline Darcy Number & Prandtl Number & Rayleigh Number & $\begin{array}{c}\left(\mathrm{T}_{\mathrm{s}}-\mathrm{T}_{\mathrm{f}}\right)_{\max } \\
\text { at } \eta=0.5\end{array}$ & $\left(\mathrm{~T}_{\mathrm{s}}-\mathrm{T}_{\mathrm{f}}\right)_{\max }$ \\
\hline \multirow[t]{4}{*}{$10^{-2}$} & 1 & $10^{6}$ & 0.000865 & 0.0248 \\
\hline & & $10^{8}$ & 0.0429 & 0.1506 \\
\hline & 100 & $10^{6}$ & 0.0081 & 0.08385 \\
\hline & & $10^{8}$ & 0.1638 & 0.3758 \\
\hline \multirow{5}{*}{$10^{-3}$} & 1 & $10^{6}$ & 0.00004 & 0.0041 \\
\hline & & $10^{8}$ & 0.00087 & 0.02635 \\
\hline & 10 & $10^{6}$ & 0.00005 & 0.0064 \\
\hline & 100 & $10^{6}$ & 0.000075 & 0.0091 \\
\hline & & $10^{8}$ & 0.0081 & 0.0884 \\
\hline
\end{tabular}

(b)

${ }^{1}$ Nondimensional time.

maximum temperature difference is expected to occur at the edge of the thermal boundary layer, with a value $\Delta T^{*} \sim \mathrm{O}(0.5)$ at the mid-height $(\eta=0.5)$ of the domain for high Rayleigh numbers. At $\eta=0$ and 1 , the maximum temperature difference is $\sim \mathrm{O}(1)$ for high $\mathrm{Ra}$ as expected.

The next case considered is one in which the solid and fluid exchange energy in a steady-state mode through inter-phase transfer $\left(\mathrm{Nu}_{f} \neq 0\right)$. Figure $6(a)$ shows the dimensionless solid-to-fluid temperature difference $T_{s}^{*}-T_{f}^{*}$ at mid-height $(\eta=0.5)$ for several Rayleigh numbers, for $\mathrm{Da}=10^{-2}, \lambda=10^{3}, \Omega=1$, and $d / H$ $=0.0135$. Figure $6(b)$ shows dimensionless solid and fluid temperature variations at mid-height for $\operatorname{Pr}=1$. The effect of the interphase heat transfer coefficient is to lower the solid-to-fluid temperature difference. For low Rayleigh numbers $\left(\mathrm{Ra}<10^{6}\right)$, the maximum temperature difference is seen to be $\sim \mathrm{O}(0)$ as explained previously. The solid-to-fluid temperature difference increases with increasing $\mathrm{Ra}$, but is always less than that in the $\mathrm{Nu}_{f}=0$ case, i.e., less than $\mathrm{O}(0.5)$. Indeed, for $\mathrm{Ra}=10^{9}$ a maximum temperature difference of about $10 \%$ is seen. Comparing Fig. 6(b) with Fig. 5 it can be seen that for high Ra, the dimensionless solid temperature variation at the mid-height of the enclosure $(\eta=0.5)$ is not linear but follows the fluid temperature variation due to inter-phase heat exchange between solid and fluid phases.

Figure 6(a) also shows that for a Rayleigh number of $10^{6}$, increasing the Prandtl number from $\operatorname{Pr}=1$ to $\operatorname{Pr}=100$ increases the solid-to-fluid temperature difference by a small extent. The vertical velocity in the rectangular domain scales as [27]

$$
V=\frac{v d}{\alpha_{f}} \frac{H}{d}=\operatorname{Re} \operatorname{Pr}\left(\frac{H}{d}\right) \sim\left(\operatorname{RaDa}^{2}\right)^{1 / 2}
$$

The inter-phase heat transfer coefficient scales as

$$
\begin{aligned}
\mathrm{Nu}_{p} & =\frac{h_{v} d^{2}}{k_{f}}=0.376(\mathrm{Re})^{0.644} \operatorname{Pr}^{0.37} \\
& \sim 0.376\left\{\left(\operatorname{RaDa}^{2}\right)^{1 / 2}\left(\frac{d}{H}\right)\right\}^{0.644} \operatorname{Pr}^{-0.274}
\end{aligned}
$$

Thus, the inter-phase Nusselt number depends on the Prandtl number, and tends to decrease as the Prandtl number increases, causing the two media to act in a more uncoupled manner. This dependence assumes that the Prandtl number variation in Eq. (6) is valid at high Pr.

Unsteady Flow. As in the steady-state cases, in order to understand the effect of the inter-phase heat transfer on the solid-tofluid temperature difference we first consider the $\mathrm{Nu}_{f}=0$ case. In the absence of inter-phase heat exchange, the solid-to-fluid temperature difference depends on the relative response time of the two phases. The solid reaches steady state in time scales of order $\left(H^{2} / \alpha_{s}\right)$ or a dimensionless time scale of $\tau_{s} \sim \Omega / \lambda$. The fluid response time depends on Ra. For low Rayleigh numbers the time to reach steady state scales as $H^{2} / \alpha_{f}$, corresponding to a dimensionless scale $\tau_{f} \sim \mathrm{O}(1)$. For high Rayleigh numbers, the fluid time-to-steady-state scales as the inverse of the vertical velocity, and, hence,

$$
\begin{gathered}
V \sim\left(\mathrm{RaDa}^{2}\right)^{1 / 2} \\
\tau_{f} \sim 1 / V \sim\left(\mathrm{RaDa}^{2}\right)^{-1 / 2}
\end{gathered}
$$

Figure $7(a)$ shows the variation of dimensionless solid-to-fluid temperature difference along $\eta=0.5$ at different times for $\mathrm{Ra}$ $=10^{6}, \mathrm{Da}=10^{-2}, \operatorname{Pr}=1, \Omega=1000$, and $\lambda=1$. Figure $7(b)$ shows the fluid and solid temperature distributions at the mid-height of the domain. The solid response time is much faster than that of the fluid due to its higher thermal diffusivity, and it is seen to reach a steady state much faster. In Fig. 7(b), the solid has reached a steady state even before the fluid field starts to develop. Thus, at 


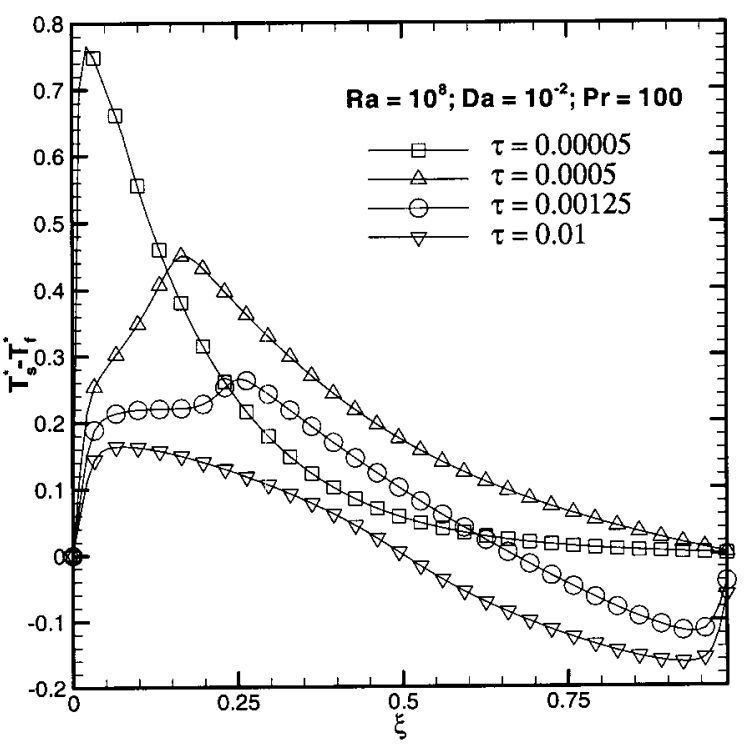

(a)

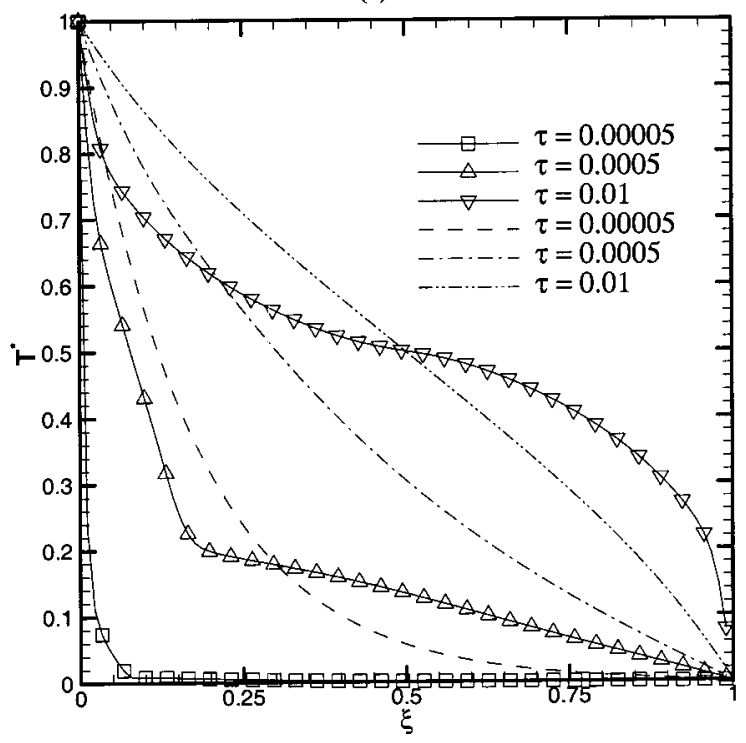

(b)

Fig. 11 Predicted temporal evolution of thermal field for Ra $=10^{8}, \mathrm{Nu}_{f} \neq 0, \mathrm{Pr}=100$, and $\mathrm{Da}=10^{-2}$ at the mid-height of the domain $(\eta=0.5)$ : (a) solid-to-fluid temperature difference, and (b) solid (broken line) and fluid (solid lines with symbols) temperature distribution

early times, the solid-to-fluid temperature difference is high $(\sim O(1))$ and the temperature difference progressively decreases with time, reaching its steady-state value over the fluid time scale. The ratio of the solid-to-fluid time to reach steady state is given by

$$
\frac{\tau_{s}}{\tau_{f}} \sim \frac{\Omega\left(\mathrm{RaDa}^{2}\right)^{1 / 2}}{\lambda}
$$

As the Rayleigh number increases, the fluid response time decreases. This is seen in Fig. 8(a) which shows a plot of temperature difference variation along the mid-height of the domain for $\mathrm{Ra}=10^{8}, \mathrm{Da}=10^{-2}, \mathrm{Pr}=1$, and $\mathrm{Nu}_{f}=0$. Figure $8(b)$ shows the variation of solid and fluid temperature fields along the mid-height of the domain. Due to the high Rayleigh number, the core fluid is at a temperature of $T_{f}^{*}=0.5$ and the boundary layer is confined to a very thin layer near the heated and cooled walls. The time to reach a steady state and the solid-to-fluid temperature difference at steady state are listed in Tables 1(a) and 1(b) for various Darcy,

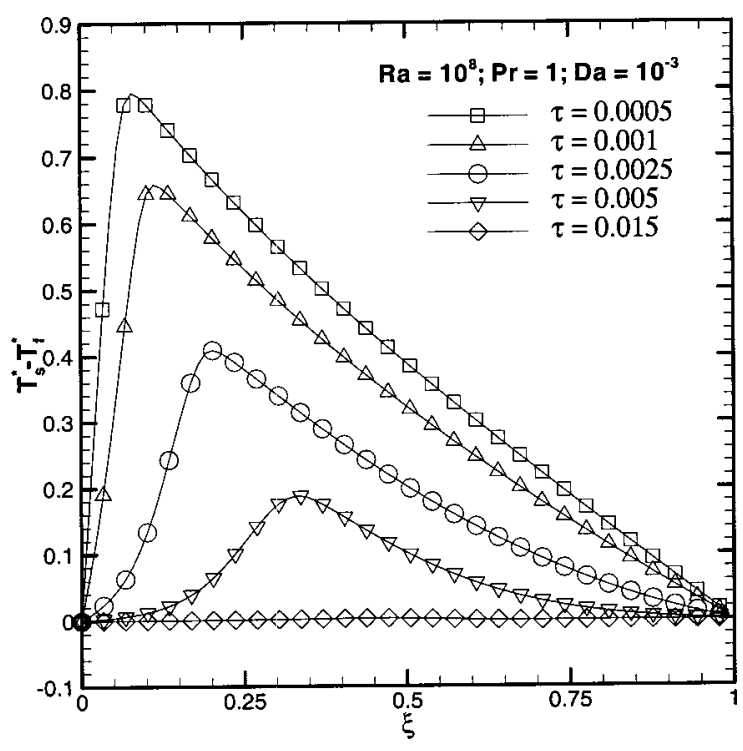

Fig. 12 Predicted temporal evolution of solid-to-fluid temperature difference at the mid-height of the domain $(\eta=0.5)$ for $\mathbf{R a}$ $=10^{8}, \mathrm{Nu}_{f} \neq 0, \mathrm{Pr}=1$, and $\mathrm{Da}=10^{-3}$

Prandtl and Rayleigh numbers. The process is declared to have reached a steady state if $T^{*}$ at $(\eta, \xi)=(0.78,0.98)$ is within $1 \%$ of its steady-state value. It is clear from Tables 1(a) and 1(b) that for $\mathrm{Nu}_{f}=0$, the time to reach steady state is independent of Pr, as expected. For low Darcy number $\left(\mathrm{Da}=10^{-3}\right)$, the fluid flow is so strongly retarded by the porous medium that the time to reach a steady-state is governed by the fluid diffusion scale, $\tau_{f} \sim \mathrm{O}(1)$; correspondingly, the solid-to-fluid temperature difference is relatively low.

The results for $\mathrm{Nu}_{f} \neq 0$ are considered next. Here, the behavior is governed by the relative magnitude of three interacting scales: the response time of the solid, the response time of the fluid, and the response time of the solid-fluid interface. If the interface response time is very long, the solid and fluid evolve separately and large temperature differences exist at steady state. If, on the other hand, the interface response time is much shorter than either the solid or the fluid response time, the two phases will evolve in a coupled manner, with the faster-responding phase imprinting its temperature on the slower-responding phase. A measure of the dimensionless response time of the interface is $\mathrm{Nu}_{f}^{-1}$ $\left(=h_{v} H^{2} / k_{f}\right)^{-1}$. For $\mathrm{Nu}_{f} \gg 1$, the two phases will evolve in equilibrium. Figure $9(a)$ shows the temporal evolution of the solid-tofluid temperature difference for a Rayleigh number of $10^{6}, \operatorname{Pr}=1$ and $\mathrm{Da}=10^{-2}$. The dimensionless solid and fluid temperature distribution at $\eta=0.5$ is shown in Fig. $9(b)$. The solid has the fastest response time, and reaches a steady state on a time scale $\tau_{s}$ $\sim \Omega / \lambda$. The fluid time scale for this low value of $\operatorname{RaDa}^{2}(=100)$ is relatively large, leading to a slow approach to steady state. Thus, the fluid is essentially at its initial condition during the time the solid reaches a steady state, and initial temperature differences in Fig. $9(a)$ are $O(1)$. However, it is seen that the solid-to-fluid temperature difference is driven to zero over a time scale $\tau \sim \mathrm{O}(0.015)$, far faster than the response time of the fluid. This is a result of the response time of the inter-phase heat transfer, which is intermediate between the solid and fluid scales. This allows the solid to impose its imprint on the fluid temperature profile, which also assumes a straight line shape, leading to nearly zero temperature difference at steady state. For the uncoupled case (Fig. 7(b)), the fluid temperature is not a straight line for the same set of parameters.

In contrast, the interface time-scale is competitive with the solid time scale in Figs. 10(a) and 10(b), which shows the solid-to-fluid 
temperature difference and the solid and fluid temperature variation at mid-height for $\mathrm{Ra}=10^{8}, \mathrm{Pr}=1$, and $\mathrm{Da}=10^{-2}$. The ratio of the interface time scale to the solid response time is $\sim\left(\mathrm{Nu}_{f} \Omega / \lambda\right)^{-1}$. Here, the solid is not allowed to reach a steady state before losing heat to the fluid. Instead, the temperature difference begins to fall well before $\tau \sim \tau_{s}$ and reaches equilibrium with the fluid on a time scale of $\tau \sim 0.0025$. Because the fluid time scale is more competitive with the solid for this high value of $\operatorname{RaDa}^{2}\left(=10^{4}\right)$, we see that the solid temperature profile is not imposed on the fluid and a nonzero temperature difference exists at steady state. The time to reach steady-state and the steady state solid-to-fluid temperature difference for several $\mathrm{Ra}, \mathrm{Pr}$, and $\mathrm{Da}$ are listed in Tables 2(a) and 2(b), respectively. Since the two phases are coupled, the time to reach steady state is of the same order for both. This is especially true as $\mathrm{Ra}$ and $\mathrm{Da}$ increase, since $\mathrm{Nu}_{p}$ increases with $\mathrm{RaDa}^{2}$.

The effect of Prandtl number on the solid-to-fluid temperature difference and the temporal evolution of the solid and fluid temperature fields is brought out in Figs. 11(a) and 11(b) for which $\mathrm{Ra}=10^{8}, \operatorname{Pr}=100$, and $\mathrm{Da}=10^{-2}$. From Eq. (2), it can be seen that an increase in Prandtl number causes a decrease in the interphase heat transfer coefficient, and, hence, the phases act in a more uncoupled manner. The temporal evolution is faster than the uncoupled case (Figs. $8(a)$ and $8(b)$ ) and the solid-to-fluid temperature difference is higher than the corresponding $\operatorname{Pr}=1$ and $\mathrm{Nu}_{f} \neq 0$ case. It is important to note, however, that these results are subject to the assumption that Eq. (6) describes the inter-phase heat transfer.

The effect of Darcy number can be seen by comparing Fig. 12 with Fig. 9. Figure 9 has been plotted for $\mathrm{Ra}=10^{6}, \mathrm{Da}=10^{-2}$ while Fig. 12 has been plotted for $\mathrm{Ra}=10^{8}, \mathrm{Da}=10^{-3}$. The fluid velocity scales approximately as $\sim\left(\mathrm{RaDa}^{2}\right)^{1 / 2}$. As a result, the temperatures differences in the two figures are almost identical; the response times are also very similar.

\section{Conclusions}

A fully transient analysis of natural convection inside a porous enclosure containing a metal foam was carried out using a twotemperature formulation. Based on the results of a parametric study, local thermal equilibrium is not ensured, either during the transient or at steady state in such systems. For transient applications, the temperature difference between the solid and the fluid is governed by the response time of the two phases as well as the time scale of the solid-fluid interface. The results demonstrate that even if the steady-state temperature differences are small, the temperature difference during the unsteady response may be quite large. Single-temperature formulations are expected to yield incorrect predictions when the inter-phase heat transfer time scale is longer than the fastest response time of either the fluid or solid phase. For metal/air or metal/PCM systems, the faster-responding phase for typical ranges of $\mathrm{Ra}$ and $\mathrm{Da}$ is the solid. In this limit, two-temperature formulations are necessary when $\mathrm{Nu}_{f}<1$.

The present study did not consider phase change. When phase change is included, an additional time scale associated with the latent heat of melting is introduced in the problem, and depending on the value of the Stefan number, can change the results obtained here. Yet another time scale appears when pulsed heating is used. The interaction of pulse periodicity with these competing scales determines the validity of single-temperature formulations. These effects will be investigated in the future. Another research need is the development of interphase Nusselt number correlations for high Prandtl number and low-Reynolds number flows, as well as correlations for pure conduction heat transfer in PCMimpregnated foams. These correlations are necessary to correctly capture inter-phase heat transfer in the frozen as well as the lowvelocity sections of the computational domain.

\section{Nomenclature}

$a_{s f}=$ Ratio of specific surface area to volume, $\mathrm{m}^{-1}$

$C_{p}=$ Specific heat, $\mathrm{J} \mathrm{kg}^{-1} \mathrm{~K}^{-1}$

$\mathrm{Da}=$ Darcy number

$\mathrm{d}=$ Particle diameter or mean pore diameter, $\mathrm{m}$

$F=$ Inertial coefficient

$g=$ Acceleration due to gravity, $\mathrm{ms}^{-2}$

$H=$ Height of enclosure, $\mathrm{m}$

$h_{s f}=$ Inter-phase heat transfer coefficient, $\mathrm{W} \mathrm{m}^{-2} \mathrm{~K}^{-1}$

$h_{v}=$ Volumetric heat transfer coefficient, $\mathrm{W} \mathrm{m}^{-3} \mathrm{~K}^{-1}$

$K=$ Permeability, $\mathrm{m}^{2}$

$k=$ Thermal conductivity, $\mathrm{W} \mathrm{m}^{-1} \mathrm{~K}^{-1}$

$\mathrm{Nu}=$ Nusselt number based on height of the enclosure

$P=$ Pressure, $\mathrm{N} \mathrm{m}^{-2}$

Pr $=$ Prandtl number

$\mathrm{Ra}=$ Rayleigh number

$\mathrm{Re}=$ Reynolds number

$T=$ Temperature, $\mathrm{K}$

$t=$ Time, $\mathrm{s}$

$U=$ Velocity vector, $\mathrm{ms}^{-1}$

$u, v=$ Velocity in $\mathrm{x}$ and $\mathrm{y}$ directions, $\mathrm{ms}^{-1}$

$x, y=$ Cartesian coordinates

\section{Greek Symbols}

$\alpha=$ Thermal diffusivity, $\mathrm{m}^{2} \mathrm{~s}^{-1}$

$\beta=$ Thermal expansion coefficient, $\mathrm{K}^{-1}$

$\Delta H=$ Enthalpy of freezing/melting, $\mathrm{J} \mathrm{kg}^{-1}$

$\Delta t=$ Time step

$\Delta x, \Delta y=$ Spatial mesh sizes, $\mathrm{m}$

$\delta=$ Ratio of mean pore diameter to height of enclosure

$\varepsilon=$ Porosity

$\eta=$ Dimensionless y coordinate

$\lambda=$ Ratio of thermal conductivities of solid and fluid

$\mu=$ Dynamic viscosity, $\mathrm{N} \mathrm{s} \mathrm{m}^{-2}$

$\nu=$ Kinematic viscosity, $\mathrm{m}^{2} \mathrm{~s}^{-1}$

$\rho=$ Density, $\mathrm{kg} \mathrm{m}^{-3}$

$\tau=$ Dimensionless time

$\xi=$ Dimensionless $\mathrm{x}$ coordinate

$\Omega=$ ratio of volumetric heat capacities of solid and liquid

\section{Subscripts}

$c=$ cold

ef $=$ effective fluid property

$e s=$ effective solid property

$f=$ fluid

$h=$ hot

$l=$ liquid

$p=$ pore or particle

$s=$ solid

\section{Superscripts}

$*$ dimensionless quantity

$s s=$ steady state

\section{Acknowledgment}

Support for this work from industry members of the Cooling Technologies Research Center, an NSF Industry/University Cooperative Research Center (www.ecn.purdue.edu/CTRC), is gratefully acknowledged.

\section{References}

[1] Himran, S., Suwono, A., and Mansoori, G. A., 1994, "Characterization of Alkanes and Paraffin Waxes for Application as Phase Change Energy Storage Medium," Energy Sources, 16, pp. 117-128.

[2] Krishnan, S., 2002, "Analysis of Phase Change Energy Storage Systems for Pulsed Power Dissipation,” M.S.M.E. thesis, Purdue University, West Lafayette, IN.

[3] Krishnan, S., Murthy, J. Y., and Garimella, S. V., 2002, “A Two-Temperature Model for the Analysis of Passive Thermal Control Systems for Electronics," Proc. of IMECE 2002, ASME Paper No. 33335, ASME, New York.

[4] Hunt, M. L., and Tien, C. L., 1988, "Effects of Thermal Dispersion on Forced 
Convection in Fibrous Media,” Int. J. Heat Mass Transfer, 31(2), pp. 301-309.

[5] Amiri, A., and Vafai, K., 1994, "Analysis of Dispersion Effects and NonThermal Equilibrium, Non-Darcian, Variable Porosity Incompressible Flow Through Porous Media," Int. J. Heat Mass Transfer, 37(6), pp. 939-954.

[6] Amiri, A., and Vafai, K., 1998, "Transient Analysis of Incompressible Flow Through a Packed Bed," Int. J. Heat Mass Transfer, 41(24), pp. 4259-4279.

[7] Lee, D. Y., and Vafai, K., 1999, "Analytical Characterization and Conceptual Assessment of Solid and Fluid Temperature Differentials in Porous Media," Int. J. Heat Mass Transfer, 42(3), pp. 423-435.

[8] Lu, T. J., Stone, H. A., and Ashby, M. F., 1998, "Heat Transfer in Open-Cell Metal Foams," Acta Mater., 46(10), pp. 3619-3635.

[9] Minkowycz, W. J., Haji-Sheikh, A., and Vafai, K., 1999, “On Departure from Local Thermal Equilibrium in Porous Media due to a Rapidly Changing Heat Source: the Sparrow Number," Int. J. Heat Mass Transfer, 42(18), pp. 3373 3385 .

[10] Calmidi, V. V., and Mahajan, R. L., 1999, "The Effective Thermal Conductivity of High Porosity Fibrous Metal Foams," ASME J. Heat Transfer, 121, pp. $466-471$.

[11] Alazmi, B., and Vafai, K., 2000, "Analysis of Variants Within the Porous Media Transport Models," ASME J. Heat Transfer, 122, pp. 303-326.

[12] Calmidi, V. V., and Mahajan, R. L., 2000, "Forced Convection in High Porosity Metal Foams," ASME J. Heat Transfer, 122, pp. 557-565.

[13] Hwang, J. J., Hwang, G. J., Yeh, R. H., and Chao, C. H., 2002, “Measurement of Interstitial Convective Heat Transfer and Frictional Drag for Flow Across Metal Foams," ASME J. Heat Transfer, 124, pp. 120-129.

[14] Kuznetsov, A. V., 1998, "Thermal Nonequilibrium Forced Convection in Porous Media," Transport Phenomena in Porous Media, Ingham, D. B., and Pop, I., eds., Elsevier, Oxford, pp. 103-129.

[15] Nield, D. A., and Bejan, A., 1992, Convection in Porous Media, 1st ed. Springer-Verlag, New York, NY.
[16] Kaviany, M., 1995, Principles of Heat Transfer in Porous Media, 2nd ed. Springer-Verlag, New York, NY.

[17] Deiber, J. A., and Bortolozzi, R. A., 1998, “A Two-Field Model for Natural Convection in a Porous Annulus at High Rayleigh Numbers," Chem. Eng. Sci., 53, pp. 1505-1516.

[18] Mohammed, A. A., 2000, "Non-Equilibrium Natural Convection in a Differentially Heated Cavity Filled With a Saturated Porous Matrix," ASME J. Heat Transfer, 122, pp. 380-384.

[19] Rees, D. A. S., and Pop, I., 2000, "Vertical Thermal Boundary Layer Flow in a Porous Medium Using a Thermal Non-Equilibrium Model," J. Porous Media, 3, pp. 31-44.

[20] Beckermann, C., and Viskanta, R., 1988, "Natural Convection Solid/Liquid Phase Change in Porous Media," Int. J. Heat Mass Transfer, 31(1), pp. 35-46.

[21] Phanikumar, M. S., and Mahajan, R. L., 2002, "Non-Darcy Natural Convection in High Porosity Metal Foams," Int. J. Heat Mass Transfer, 45(18), pp. 3781-3793.

[22] Moyne, C., 1997, "Two-Equation Model for a Diffusive Process in Porous Media Using the Volume Averaging Method With an Unsteady-State Closure," Adv. Water Resour., 20, pp. 63-76.

[23] Wakao, N., and Kaguei, S., 1982, Heat and Mass Transfer in Packed Beds, Gordon and Beach, New York, NY.

[24] Khosla, P. K., and Rubin, S. G., “A Diagonally Dominant Second-Order Accurate Implicit Scheme," Comput. Fluids, 2, pp. 207-209.

[25] Ferziger, J. H., and Peric, M., 1995, Computational Methods for Fluid Dynamics, Springer-Verlag, Berlin.

[26] Simpson, J. E., Garimella, S. V., and deGroh, III, H. C., 2002, “An Experimental and Numerical Investigation of Bridgman Growth of Succinonitrile," AIAA J. Thermophysics and Heat Transfer, 16(3), pp. 324-335.

[27] Bejan, A., 1995, Convection Heat Transfer, 2nd ed., John Wiley and Sons, New York, NY. 